\title{
DESENVOLVIMENTO, PRODUÇÃO E CARACTERIZAÇÃo DE NANOCRISTAIS DE FÁRMACOS POUCO SOLÚVEIS
}

\section{Joana F. Fangueiro e Inês R. Marques}

Faculdade de Ciências da Saúde, Universidade Fernando Pessoa, 4200-150 Porto, Portugal

Patrícia Severino

Departamento de Processos Biotecnológicos, Faculdade de Engenharia Química, Universidade Estadual de Campinas, 13083-852 Campinas - SP, Brasil / Faculdade de Ciências da Saúde, Universidade Fernando Pessoa, 4200-150 Porto, Portugal

\section{Maria Helena A. Santana}

Departamento de Processos Biotecnológicos, Faculdade de Engenharia Química, Universidade Estadual de Campinas, 13083-852

Campinas - SP, Brasil

Eliana B. Souto*

Faculdade de Ciências da Saúde, Universidade Fernando Pessoa, 4200-150 Porto, Portugal / Instituto de Biotecnologia e Bioengenharia, Centro de Genómica e Biotecnologia, Universidade de Trás-os-Montes e Alto Douro, 5001-801 Vila Real, Portugal

Recebido em 15/12/11; aceito em 24/4/12; publicado na web em 10/8/12

\begin{abstract}
DEVELOPMENT, PRODUCTION AND CHARACTERIZATION OF NANOCRYSTALS OF POORLY SOLUBLE DRUGS. Poorly soluble drugs have low bioavailability, representing a major challenge for the pharmaceutical industry. Processing drugs into the nanosized range changes their physical properties, and these are being used in pharmaceutics to develop innovative formulations known as Nanocrystals. Use of nanocrystals to overcome the problem of low bioavailability, and their production using different techniques such as microfluidization or high pressure homogenization, was reviewed in this paper. Examples of drugs, cosmetics and nutraceutical ingredients were also discussed. These technologies are well established in the pharmaceutical industry and are approved by the Food and Drug Administration.
\end{abstract}

Keywords: nancrystals; microfluidization; high pressure homogenization.

\section{INTRODUÇÃO}

O desenvolvimento da biotecnologia em paralelo com a tecnologia farmacêutica tem fornecido informações extremamente úteis para o desenvolvimento de novas formas farmacêuticas. Contudo, cerca de $40 \%$ de novos fármacos produzidos apresentam problemas de baixa biodisponibilidade causada, principalmente, pela sua insolubilidade em fluidos biológicos. São exemplos a afidicolina, a violaceína e o Proteic - Magnesium Ammonium Phospholinoleate Anhydride ou P-MAPA (Figura 1). ${ }^{1,2}$

A biodisponibilidade do fármaco é influenciada por diversos fatores como a velocidade de dissolução, tamanho da partícula, área superficial, polimorfismo do fármaco, formação de sais, permeabilidade, lipofilia, pKa. ${ }^{3,4}$ No caso de fármacos insolúveis, a biodisponibilidade pode ser melhorada através da micronização da matéria-prima, que promove o aumento da sua área superficial. No entanto, para certas substâncias este procedimento não é suficiente, como é o caso do itraconazol, ${ }^{5}$ o acetato de megestrol, ${ }^{6}$ ou o paclitaxel. ${ }^{7}$ Face a esta realidade, surge a necessidade de transformação desses fármacos em estruturas do tipo nanocristal. ${ }^{8,9}$ As vantagens deste procedimento incluem aumento da velocidade de dissolução e saturação da solubilidade, reprodutibilidade da absorção oral e liberação controlada, proporcionando a redução do número de doses. ${ }^{10,11}$ Essa tecnologia também é útil para fármacos administrados por via oral que apresentam absorção limitada, para os fármacos administrados por via intravenosa na forma de suspensão ${ }^{12} \mathrm{e}$ àqueles que pertencem às classes II e IV do Sistema de Classificação Biofarmacêutico. ${ }^{13} \mathrm{O}$ aumento da área superficial dos nanocristais de fármacos induz, consequentemente, $\mathrm{o}$ aumento da absorção oral e, simultaneamente, a sua solubilidade de

*e-mail: eliana@ufp.edu.pt<smiles>CC12C[C@H](O)[C@H](CO)C[C@H]1CC[C@@H]1[C@H](CO)[C@H](O)CC[C@@]12C</smiles><smiles>O=C1NC(c2c[nH]c3ccc(Cl)cc23)=C/C1=C1\C(=O)Nc2ccccc21</smiles>

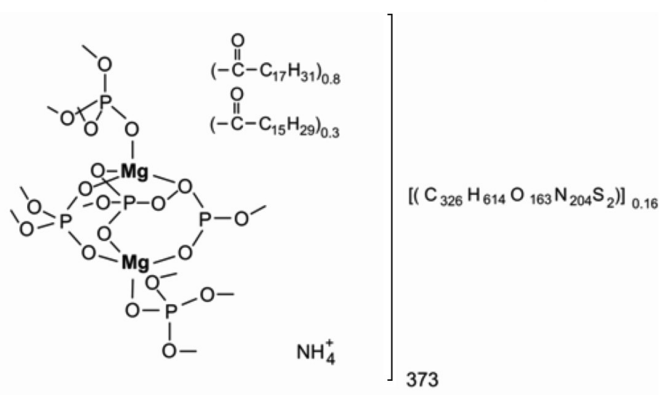

Figura 1. Estrutura das moléculas (a) afidicolina; (b) violaceína; (c) P-MAPA. Adaptada das refs. 1 e 2

saturação. ${ }^{14}$ Esta tecnologia é tão promissora que em menos de 10 anos de estudos, a primeira patente foi depositada em $1990,{ }^{15} \mathrm{com}$ o primeiro produto lançado no mercado em 2000, o Emend ${ }^{\circledR}$. Este curto período de tempo confirma que é um procedimento industrial 
viável. ${ }^{16,17} \mathrm{O}$ aprepitando é o fármaco do Emend ${ }^{\circledR}$ utilizado para o alívio de náuseas em tratamentos quimioterápicos. O aumento da área superficial promovido pela "nanonização" permite que esta formulação apresente maior absorção e biodisponibilidade, em comparação com a forma farmacêutica micronizada. ${ }^{18}$

\section{DEFINIÇÕES E PROPRIEDADES DOS NANOCRISTAIS DE FÁRMACOS}

Um nanocristal de fármaco caracteriza-se por ser uma partícula de dimensão menor que $100 \mathrm{~nm}$, que apresenta enorme interesse tecnológico devido às suas propriedades termodinâmicas, isto é, uma forte dependência em relação ao tamanho que pode ser controlado pelos processos de produção. Além disso, os nanocristais de fármacos apresentam um domínio único que ajuda a explicar o comportamento macroscópico de outros fármacos similares, pois as propriedades físico-químicas e termodinâmicas sofrem mudanças com a variação do tamanho. ${ }^{16}$ As vantagens incluem a elevada capacidade de carga do sistema, simplicidade da formulação, fácil transposição de escala, assim como a possibilidade da produção ser de baixo custo ${ }^{3}$ e não recorrer a solventes orgânicos..$^{19}$ Além disso, ao contrário dos fármacos micronizados, os nanocristais de fármacos podem ser administrados através de diferentes vias. A administração oral é a forma mais aceitável pelos pacientes. Com os nanocristais de fármacos, é possível administrar na forma de uma nanossuspensão ou ser transformada para formas de dosagem sólidas como, por exemplo, comprimidos, granulados ou cápsulas. ${ }^{20}$

$\mathrm{Na}$ administração oral, os nanocristais de fármacos apresentam melhoras significativas nas propriedades de absorção e biodisponibilidade. No que respeita à velocidade de dissolução dos fármacos micronizados, esta característica é otimizada pelo aumento da área de superfície, enquanto nos fármacos nanonizados apresenta um efeito ainda mais evidente, pois a área superficial é aumentada e, consequentemente, a velocidade de dissolução é maior. ${ }^{10,16}$

Outro aspecto importante em relação aos nanocristais de fármacos é o aumento da solubilidade de saturação, que é um componente específico constante que depende da temperatura e das propriedades do meio de dissolução. A solubilidade de saturação é um equilíbrio entre moléculas, aumentando-se a pressão de dissolução muda o equilíbrio e a solubilidade de saturação aumenta. $\mathrm{O}$ aumento da solubilidade de saturação e o aumento do lúmen intestinal contribuem para o aumento do gradiente de concentração entre o lúmen e o sangue, acelerando a difusão do fármaco e, por conseguinte, a absorção. ${ }^{21}$

Devido o tamanho reduzido dos nanocristais de fármacos, estes promovem a mucoadesividade, isto é a adesão às superfícies. A mucoadesividade das partículas à parede intestinal, depois da administração oral, melhora a biodisponibilidade e o fármaco dissolve-se e absorve-se exatamente no local desejado. ${ }^{17}$

Quando produzidos por homogeneização à alta pressão, a desvantagem que pode ser apontada é o número de passagem, podendo chegar a 75 ciclos, enquanto no caso da técnica de precipitação é essencial manter o tamanho dos nanocristais após o processo. Em alguns casos, a partícula cristalina se torna amorfa e essa mudança deve ser mantida por toda vida útil do produto, para evitar a alteração da biodisponibilidade. ${ }^{3}$ Além disso, deve ter-se em consideração a escolha do tensoativo, que influencia o tamanho e a estabilidade da partícula, prevenindo a agregação. Polímeros e tensoativos agem formando uma barreira superficial no nanocristal, promovendo a estabilização físico-química da formulação. ${ }^{22}$

Xia et al. ${ }^{23}$ estudaram o efeito do tamanho do cristal de nitrendipina na dissolução e absorção oral in vivo. Cinco tipos de suspensões de nanocristais de nitrendipina com tamanhos de 200 e 620 nm, 2,7; 4,1 20,2 $\mu \mathrm{m}$ foram preparados por precipitação e ultrassonicação, ou pelo método de precipitação antissolvente. Para o estudo de dissolução foi utilizado o fluido intestinal simulado em jejum e a biodisponibilidade in vivo foi realizada com ratos Wistar. Os resultados mostraram que a taxa de dissolução in vitro e de biodisponibilidade in vivo da nitrendipina foi significativamente maior com a redução do tamanho das partículas.

$\mathrm{Na}$ administração tópica observa-se que os nanocristais de fármacos não penetram diretamente na epiderme, mas através dos folículos pilosos. Tal se explica pelo aumento da solubilidade de saturação, levando a um aumento do gradiente de concentração e, posteriormente, a maior penetração na pele. Além disso, as moléculas lipofílicas penetram melhor do que as hidrofílicas. Esta explicação justifica o facto dos nanocristais de rutina apresentarem absorção superior, quando comparados com a rutina hidrofílica. ${ }^{17,24}$

Outra via promissora para a administração de nanocristais de fármacos é a intravenosa, pois proporciona uma biodisponibilidade de $100 \%$. Gao et al..$^{25}$ estudaram a produção de nanocristais de curcumina para uso injetável, com o objetivo de diminuir a citotoxicidade. A nanossuspensão foi preparada por homogeneização à alta pressão, avaliando-se morfologia, tamanho, potencial zeta, solubilidade, taxa de dissolução e cristalinidade dos nanocristais obtidos. Os nanocristais de curcumina apresentaram forma esfera, com diâmetro médio de $250,6 \mathrm{~nm}$ e potencial zeta de $-27,92 \mathrm{mV}$. A solubilidade e a taxa de dissolução aumentaram significativamente, devido ao pequeno tamanho de partículas, e o estado cristalino do fármaco foi preservado para aumentar sua estabilidade contra a degradação. A avaliação do risco de efeitos secundários revelou que os nanocristais de curcumina promovem menor irritação local, menor riscos de flebite e menor taxa de hemólise das hemácias, em comparação com o fármaco puro. Estes resultados mostram que os nanocristais de curcumina representam uma forma promissora de administração de fármacos para o tratamento do câncer.

Vários relatos demonstraram que os nanocristais apresentam efeitos semelhantes aos das nanopartículas transportadoras após a administração intravenosa, resultando em captação pelo sistema fagocítico mononuclear (e.g. fígado, baço, pulmão e medula óssea). Assim, os nanocristais são benéficos para o tratamento de doenças causadas por parasitas, que residem nos macrófagos do sistema fagocítico mononuclear, e para o tratamento de câncer nos órgãos acima mencionados. ${ }^{11}$

Lou et al. estudaram a citoxicidade in vitro e a ação antitumoral in vivo dos nanocristais de oridonin, obtendo partículas de tamanho $897,2 \pm 14,2 \mathrm{~nm}$ e potencial zeta de $-21,8 \pm 0,8 \mathrm{mV}$. Os resultados mostraram que a formulação induz maior apoptose, em comparação com o fármaco livre e os resultados in vivo apresentaram maior eficácia na ação antitumoral, com redução do volume e peso do tumor, sugerindo que a formulação em estudo é promissora para o tratamento de tumores. ${ }^{26}$

\section{MÉTODOS DE PRODUÇÃO}

Os métodos de preparação dos nanocristais de fármacos são divididos em Top down e Bottom up. Na técnica de Bottom up, o fármaco é dissolvido em solvente e um cossolvente e, em seguida, é realizada a precipitação. A produção dos nanocristais de fármacos por Top down é semelhante à produção de nanoemulsões, sendo as técnicas mais utilizadas a microfluidização ${ }^{19} \mathrm{e}$ a homogeneização à alta pressão. ${ }^{20,27}$ Nestas técnicas, a preparação da nanoemulsão ocorre em duas etapas. Na primeira, as fases aquosa e oleosa são aquecidas separadamente e emulsificadas através de um homogeneizador de alta velocidade (Ultra-Turrax ${ }^{\circledR}$ ), sendo formada uma emulsão primária, com partículas de tamanho micrométrico. Na segunda etapa, o diâmetro das partículas é progressivamente reduzido a valores nanométricos 
(100-300 nm), através de um microfluizador ou por homogeneização à alta pressão. ${ }^{28}$ A produção dos nanocristais de fármacos é possível devido à interação entre a superfície da partícula e o solvente, por intermédio de um tensoativo ou polímero, superando as diferenças de densidade. ${ }^{16} \mathrm{~A}$ produção por Top down é um processo fácil, rápido, escalonáve ${ }^{20}$ e de baixo custo, tornando-se uma atrativa metodologia para resolver o problema de insolubilidade, biodiodisponibilidade e absorção de princípios ativos pouco hidrossolúveis. ${ }^{22}$ Apresenta, contudo, a desvantagem de ser uma técnica que demanda grande quantidade de energia e experiência. ${ }^{29}$

\section{Microfluidização}

A divulgação da técnica de microfluidização iniciou-se em 1985. ${ }^{30}$ Este método utiliza alta pressão para direcionar o fluxo de uma pré-emulsão para uma área de impacto. A área de impacto pode ser do tipo " $Z$ ", em que a suspensão muda, por minutos, a direcção do seu fluxo, levando à colisão entre partículas e forças. No segundo tipo de câmara, o tipo "Y", a corrente da suspensão é dividida em 2 correntes que colidem frontalmente. ${ }^{14}$ Essa técnica tem sido empregada pela companhia canadense SkyePharma Canada Inc (Reasearch Triangle Pharmaceuticals) para fármacos insolúveis, ${ }^{16,20}$ como o medicamento Triglide $^{\circledR}$ (fenofibrato), que está sendo comercializado para redução dos níveis de colesterol. A desvantagem é o alto número de ciclos necessários, podendo a formulação apresentar alta polidispersidade. ${ }^{20}$

\section{Homogeneização à alta pressão}

A homogeneização à alta pressão é aceita pelas autoridades reguladoras para a produção de emulsões para nutrição parenteral (e.g. Intralipid $^{\circledR}$, Lipofudin $\left.{ }^{\circledR}\right),{ }^{31}$ sendo muito favorável para estabelecer uma nova tecnologia, (e.g. Dissocubes ${ }^{\circledR}(\text { SkyePharma })^{32}$ e Nanopure $^{\circledR}$ (PharmaSol GmbH, Berlin, now Abbott Laboratories) ${ }^{33}$ ). Entre os equipamentos disponíveis no mercado, os mais utilizados são APV Gaulin, Avestin e Niro Soavi. Esta mesma tecnologia de homogeneização à alta pressão foi adquirida pela Skypharma PLC no final dos anos 90 e aplicada no desenvolvimento de suas formulações. ${ }^{14}$

Durante muitos anos, a cavitação foi considerada como o efeito mais importante para diminuir partículas por homogeneização à alta pressão. Esta técnica tem sido descrita por diversos autores. ${ }^{32,34,35} \mathrm{~A}$ suspensão é pressionada através de um orifício de 3-5 mm a uma pressão entre 150-1500 bar. De acordo com a Lei de Bernoulli, o volume do líquido num sistema fechado por amostra representativa é constante. Isto significa que a redução no diâmetro leva a um aumento na pressão dinâmica e, simultaneamente, uma diminuição da pressão estática, quando o líquido está na fenda do homogeneizador. ${ }^{32,34,35}$

Sahoo et al. ${ }^{36}$ estudaram a produção de nanocristais de quercetina por homogeneização à alta pressão para administração oral. Avaliaram o tamanho da partícula, a cristalinidade, o perfil de dissolução e o efeito antioxidante. Os resultados apresentaram tamanho de $483 \mathrm{~nm}$ utilizando 20 ciclos após homogeneização à alta pressão a 1500 bar, a difração de raios X e a calorimetria diferencial de varredura revelaram a produção de partículas cristalinas, o perfil de dissolução obtido foi muito maior, quando comparado com o fármaco puro e a atividade antioxidante foi mais efetiva na forma de nanocristais.

\section{Dissocubes $^{\circledR}$}

Esta tecnologia é baseada na redução de partículas por homogeneização em água pura, sendo o produto comercial denominado de Dissocubes ${ }^{\circledR} \mathrm{e}$ foi desenvolvida por Müller e colaboradores,${ }^{27}$ empregando o APV/Gaulin/Rannie homogeneizadores. A metodologia consiste em dispersar o fármaco em uma solução aquosa de tensoativos, seguindo-se a sua passagem pelo homogeneizador à alta pressão sob pressão de até 4000 bar. O resultado da alta velocidade de fluxo da suspensão provoca um aumento da pressão dinâmica, sendo compensado por uma redução na pressão estática inferior à pressão de vapor da fase aquosa, fazendo com que a água comece a ferver formando bolhas de gás. Estas bolhas de gás entram em colapso imediatamente quando o líquido sai do homogeneizador à alta pressão. As partículas do fármaco são reduzidas devido ao choque causado pela cavitação. O tamanho médio das partículas no processo de homogeneização à alta pressão depende do tipo de homogeneizador, pressão, número de ciclos e dureza dos fármacos. Devido à natureza cristalina, aparece em formato cuboide ou irregular, em contraste com nanopartículas esféricas do fármaco amorfo. ${ }^{20}$

Esta tecnologia também foi utilizada por Wang et al. ${ }^{32}$ para a produção de nanocristais de deacety mycoepoxydiene, um novo fármaco empregado para o tratamento do câncer de próstata, para melhorar a solubilidade e dissolução. A formulação foi desenvolvida por homogeneização à alta pressão (DissoCubes ${ }^{\circledR}$ ) e transformado em pó, por liofilização. A nanossuspensão produzida foi avaliada por análise da morfologia, tamanho, potencial zeta, solubilidade, taxa de dissolução, cristalinidade e citotoxicidade. Os resultados mostraram que o tamanho obtido foi de $515 \pm 18 \mathrm{~nm}$ com polidispersidade de $0,12 \pm 0,03$ e potencial zeta de $-23,1 \pm 3,5 \mathrm{mV}$. No ensaio de dissolução in vitro observou-se uma velocidade de dissolução acelerada e aumento da solubilidade de saturação, e os testes de citotoxicidade in vitro evidenciaram a maior eficácia das nanossuspensões em relação ao fármaco livre. Em conjunto, estes resultados ilustram a eficiência da produção dos nanocristais de fármacos para a terapêutica do câncer.

Nesta metodologia, o uso da água pode provocar a hidrólise do fármaco e promover problemas nos passos subsequentes da formulação, como a necessidade de remoção da água. Quando empregada em fármacos de baixo ponto de fusão, o processo de secagem pode requerer técnicas dispendiosas como a liofilização, portanto, esta tecnologia é mais adequada para formulação aquosas de nanocristais. ${ }^{29}$

\section{Nanopure $^{\circledR}$}

A tecnologia Nanopure ${ }^{\circledR}$ é uma outra abordagem utilizando o homogeneizador à alta pressão. Esta tecnologia utiliza óleos, líquidos e sólidos (fundidos) como meio de dispersão primária, acrescidos de polietilenoglicóis ou água e, opcionalmente, realiza-se homogeneização em baixas temperaturas. Esses meios têm baixa pressão de vapor. No homogeneizador à alta pressão em temperatura ambiente, a água começa a ferver, ou seja, a pressão estática da água é reduzida a valores próximos da pressão de vapor da água a $20^{\circ} \mathrm{C}$. Por exemplo, a pressão de vapor de óleo é apenas $0,01 \mathrm{hPa}(\mathrm{mbar}=0,01) \mathrm{em}$ $20{ }^{\circ} \mathrm{C}$, ou seja, 2000 vezes menor. Portanto, quando água entra em cavitação, o óleo não entra. Mesmo sem cavitação, a diminuição de tamanho é suficiente porque as forças de cisalhamento provocam colisões e turbulências. As temperaturas baixas permitem trabalhar com fármacos termolábeis.

Esta metodologia é ideal para ser empregada em fármacos lábeis em meio aquoso, que podem ser diluídos em água para formar uma suspensão isotônica para administração intravenosa. Para a produção de formulações isentas de água, é possível o emprego de um meio de dispersão volátil, como a mistura de água e etanol, podendo ser ainda possível a obtenção de um pó finamente disperso, com dissolução mais rápida no trato gastrintestinal. ${ }^{20}$ As nanossuspensões resultantes da tecnologia Nanopure ${ }^{\circledR}$ podem ainda ser usadas para enchimento de cápsulas gelatinosas duras. ${ }^{16}$

Nanocristais de anfotericina B foram produzidos com a mistura de glicerol e água (80:20), resultando em uma formulação com excelente dispersão e sem sinais de agregação. Basicamente, a redução da quantidade de água aumenta a estabilidade química; em casos como 
este, a nanossuspensão de glicerol foi um intermediário na produção da emulsão do tipo óleo/água para administração intravenosa. ${ }^{37}$

\section{Nanoedge ${ }^{\circledR}$}

A tecnologia Nanoedge ${ }^{\circledR}$, desenvolvida pela Baxter, utiliza uma etapa de precipitação seguida de um tratamento térmico para a aplicação de alta energia, por exemplo, homogeneizador à alta pressão. ${ }^{38}$ De acordo com as reivindicações da patente, o recozimento impede o crescimento dos nanocristais precipitados, sendo o recozimento definido na presente invenção como o processo de conversão de matéria que é termodinamicamente instável para uma forma mais estável, por aplicação única ou repetida de energia (calor direto ou stress mecânico), seguido de relaxamento térmico. Esta redução de energia pode ser obtida através da conversão da forma sólida a partir de menor organização para uma estrutura cristalina mais ordenada. Alternativamente, a estabilização pode ocorrer por uma reorganização das moléculas de tensoativos na interface sólido-líquido. A desvantagem desta técnica é o uso de solventes orgânicos na etapa de precipitação. No caso da produção em larga escala, precisam ser removidas quantidades elevadas de solvente por causarem potencial toxicidade, e a remoção deve ocorrer em um processo estéril, tornando ainda mais difícil e dispendioso o processo. A Baxter utiliza essa tecnologia principalmente para produção de medicamentos injetáveis, devido ao potencial comprometimento da estabilidade da nanossuspensões em meio aquoso. ${ }^{20}$

\section{ElanCorporation ${ }^{\circledR}$}

A ElanCorporation ${ }^{\circledR}$ fabrica nanocristais para uso parenteral. As partículas são produzidas por técnicas de atrito em ambiente húmido que reduz o seu tamanho de forma significativa. As partículas obtidas são estabilizadas por aglomeração através de uma superfície de adsorção com tensoativos de estatuto GRAS (Generally Regarded As Safe). O resultado final apresenta-se sob a forma de uma solução (dispersão coloidal). Estudos comprovaram um aumento na biodisponilibidade, quando comparados com produtos já comercializados, assim como o tempo necessário para atingir a concentração terapêutica desejada. ${ }^{39,40}$ Esta tecnologia tem a vantagem de promover maior capacidade de encapsulação de fármacos, reforçando assim a concentração máxima tolerada quando comparada com outros produtos comerciais. ${ }^{3}$ A primeira formulação sólida terapêutica a ser lançada no mercado, e a ser comercializada com recurso a esta tecnologia, foi o Rapamune ${ }^{\circledR}$ (sirolimus, aprovado pela FDA em 2000). Outros exemplos fabricados por esta tecnologia incluem o TriCor $^{\circledR}$ (fenofibrato, aprovado pela FDA em 2004) e o Emend ${ }^{\circledR}$ (aprepitando, aprovado pela FDA em 2000). ${ }^{39}$

\section{Produção em larga escala}

Na produção de nanocristais de fármacos em escala laboratorial utiliza-se um volume de amostra pequeno, em torno de 3 a $40 \mathrm{~mL}$. Para a transposição de escala utiliza-se uma quantidade significativamente maior. Por isso, a produção de nanocristais de fármacos por homogeneização à alta pressão apresenta a vantagem de usar uma válvula de homogeneização e geometria idênticas ao equipamento de escala de bancada e laboratorial. Utilizando homogeneizador à alta pressão em larga escala obtém-se uma produção benéfica, pois diminuem-se os ciclos de homogeneização e pressão, favorecendo o controlo da temperatura e a obtenção de um produto com maior homogeneidade e menor tamanho. ${ }^{41}$

A companhia Baxter realizou uma linha asséptica para nanossuspensões parentéricas para fármacos citotóxicos em larga escala e a vantagem é que o próprio processo de homogeneização à alta pressão apresenta um efeito redutor não somente nos cristais, mas também na desintegração das bactérias..$^{20,39} \mathrm{Al}$ Shaal et al. ${ }^{42}$ estudaram a estabilidade das nanosuspensões do flavonoide apigeninae provaram que a formulação manteve-se estável por 6 meses, estocada a $4{ }^{\circ} \mathrm{C}$, temperatura ambiente e a $40{ }^{\circ} \mathrm{C}$.

\section{PRINCIPAIS MÉTODOS FÍSICO-QUÍMICOS DE ANÁLISE UTILIZADOS PARA CARACTERIZAÇÃO DOS NANOCRISTAIS}

Os nanocristais são caracterizados quanto ao tamanho, polidispersidade e potencial zeta através de espectroscopia de correlação de fótons. Para a sua análise morfológica é empregada a microscopia eletrônica de transmissão ${ }^{4}$ e para estudos da cristalinidade do fármaco são empregadas a difração de raios X e a calorimetria diferencial de varredura. ${ }^{43}$

Ao produzir um nanocristal, o fármaco adquire maior solubilidade de saturação quando comparado com a forma cristalina. Um exemplo clássico da literatura é o palmitato de cloranfenicol, em que a modificação I apresenta solubilidade de 0,13 enquanto a forma II apresenta solubilidade de 0,43 na forma cristalina e $1,6 \mathrm{mg} / \mathrm{mL}$ na forma amorfa. ${ }^{44}$ Portanto, é importante o estudo da forma cristalina para o desenvolvimento da forma farmacêutica. Outras técnicas úteis para a caraterização físico-química incluem a espectrofotometria de Raman, a análise de fluorescência por infravermelho e por ressonância magnética nuclear. ${ }^{14,34,43}$

Wang et al $^{45}$ desenvolveram nanocristais de paclitaxel utilizando homogeneizador à alta pressão. Após a produção, a formulação foi liofilizada, obtendo-se partículas de tamanho médio de 214,4 \pm 15,03 $\mathrm{nm}$. Resultados de calorimetria diferencial de varredura mostraram que a cristalinidade do paclitaxel foi preservada após o processo de homogeneização. Os resultados de farmacocinética em plasma de rato mostraram que a formulação de nanossuspensão exibiu uma

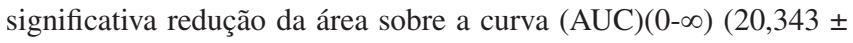
$9,119$ vs $5,196 \pm 1,426 \mu \mathrm{g} \mathrm{h} / \mathrm{mL})$, maior clearence $(2,050 \pm 0,616 \mathrm{vs}$ $0,556 \pm 0,190 \mathrm{~L} / \mathrm{kg} / \mathrm{h})$ e menor tempo de meia-vida $(5,646 \pm 2,941$ vs $3,774 \pm 1,352 \mathrm{~h}$ ), quando comparada com a solução de paclitaxel.

Li et al..$^{46}$ produziram nanocristais de cloridrato de revaprazan (inibidor de bomba protônica que apresenta baixa solubilidade e biodisponibilidade) pelo método de homogeneização à alta pressão e avaliaram a cristalinidade do fármaco em nanossuspensão e em microssuspensão, por calorimetria diferencial de varredura e difração de raios X. O fármaco manteve-se no mesmo estado cristalino, mas nos estudos de farmacocinética a formulação nanométrica apresentou maior biodisponibilidade que a formulação micrométrica.

\section{NANOCRISTAIS DE FÁRMACOS COMERCIALIZADOS}

As nanossuspensões podem ser lançadas no mercado sob a forma de produtos líquidos, especialmente para pacientes idosos e crianças. Em regra, as formas mais utilizadas para este tipo de produtos são as formas farmacêuticas sólidas, como as cápsulas e os comprimidos. No caso de nanossuspensões de fármacos em água pura (Dissocubes ${ }^{\circledR}$ ), ou em água contendo misturas (Nanopure ${ }^{\circledR}$ ), podem ser usadas como um fluído de granulação para a produção de comprimidos ou, em alternativa, como um agente molhante para a extrusão em massa da produção das grânulos. A forma sólida pode ser usada para a produção de comprimidos, grânulos ou cápsulas. Os Nanopure ${ }^{\circledR}$ podem ser produzidos em meios não aquosos, tais como óleos ou polietilenoglicóis e empregados diretamente no enchimento das cápsulas. A dispersão de cristais em óleos promove a absorção do fármaco, explorando o efeito dos lipídeos na melhoria da absorção. A Tabela 1 exemplifica os nanocristais de fármacos que estão sendo comercializados para uso oral. 
Tabela 1. Exemplos de medicamentos obtidos por nanocristais comercializados. Adaptada da ref. 16

\begin{tabular}{|c|c|c|}
\hline Nome comercial & Uso terapêutico & Empresa \\
\hline Rapamune $^{\circledast}$ & imunosupressor & Wyeth Pharmaceuticals \\
\hline Emend $^{\circledR}$ & antiemético & Merck \& Co. \\
\hline Tricor $^{\circledast}$ & hipercolesterolemia & Abbott Laboratories \\
\hline Triglide $^{\circledR}$ & hipercolesterolemia & $\begin{array}{c}\text { SkyePharma marketed; } \\
\text { Sciele Pharma }\end{array}$ \\
\hline Megace $^{\circledR}$ ES & antianorexico & $\begin{array}{l}\text { Par Pharmaceutical } \\
\text { Companies Inc. }\end{array}$ \\
\hline Avinza $^{\circledR}$ & psicoestimulante & King Pharmaceuticals \\
\hline Focalin $^{\circledR}$ & psicoestimulante & Novartis \\
\hline Ritalin $^{\circledR}$ & psicoestimulante & Novartis \\
\hline Zanaflex $^{\circledR}$ & relaxante muscular & Acorda \\
\hline
\end{tabular}

Rapamune ${ }^{\circledast}$ é um medicamento imunossupressor, muito utilizado em conjunto com a ciclosporina e esteroides, para evitar a rejeição de órgãos após o transplante de rins. Este medicamento foi introduzido no mercado em 2000 pela Wyeth Pharmaceuticals, sendo comercializado na forma de suspensão oral e comprimido. A biodisponibilidade oral da formulação é $21 \%$ maior quando comparada com a solução convencional, devido ao menor tamanho das partículas promover maior solubilidade e área superficial, consequentemente aumenta a dissolução e a biodisponibilidade. Resultados semelhantes foram obtidos com os outros medicamentos citados na Tabela 1 .

\section{CONCLUSÃO}

Os nanocristais podem ser considerados como uma abordagem universal para o desenvolvimento de formulações de fármacos insolúveis, sendo que os fármacos pouco solúveis em sistemas aquosos ainda são um grande desafio para a indústria farmacêutica. Contudo, o desenvolvimento da tecnologia de nanocristais de fármacos é promissor devido à simplicidade, eficácia, rapidez que atingiu o mercado farmacêutico e diversidade das vias de administração possíveis. Deve observar-se que a prática dessa metodologia proporciona maior solubilidade, biodisponibilidade, reprodutiblidade de doses e dissolução, cujas evidências existem de vários estudos realizados in vivo e in vitro. Os métodos de produção apresentados são simples e escalonáveis e compatíveis com as normas da FDA. Estas estratégias tecnológicas permitem abrir novos caminhos para o desenvolvimento de fármacos para serem empregues no tratamento de patologias, em menor tempo, com redução acentuada dos efeitos colaterais e, consequentemente, com maior eficácia. Geralmente, no desenvolvimento de novos fármacos obtêm-se moléculas pouco solúveis, portanto, há necessidade de formulações inteligentes para torná-las solúveis e biodisponíveis. No futuro pode almejar-se a produção de nanocristais com superfícies modificadas com o objetivo de obter uma formulação com liberação controlada.

\section{AGRADECIMENTOS}

À Fundação para a Ciência e a Tecnologia (FCT) do Ministério da Ciência e Tecnologia (PTDC/SAU-FAR/113100/2009), à Fundação de Amparo à Pesquisa do Estado de São Paulo (FAPESP/Brasil) e à Coordenação de Aperfeiçoamento de Pessoal de Nível Superior (Capes, Brasil).

\section{REFERÊNCIAS}

1. Durán, N.; Durán, M.; Tasic, L.; Marcato, P.; Quim. Nova 2010, 33, 151.
2. Merisko-Liversidge, E. M.; Liversidge, G. G.; Toxicol. Pathol. 2008, 36, 43.

3. Junghanns, J. U.; Müller, R. H.; Int. J. Nanomedicine 2008, 3, 295.

4. Miao, X.; Sun, C.; Jiang, T.; Zheng, L.; Wang, T.; Wang, S.; J. Pharm. Sci. 2011, 14, 196.

5. Sun, W.; Mao, S.; Shi, Y.; Li, L. C.; Fang, L.; J. Pharm. Sci. 2011, 100, 3365 .

6. Sylvestre, J. P.; Tang, M. C.; Furtos, A.; Leclair, G.; Meunier, M.; Leroux, J. C.; J. Control. Release 2011, 149, 273.

7. Deng, J.; Huang, L.; Liu, F.; Int. J. Pharm. 2010, 390, 242.

8. Ning, X.; Sun, J.; Han, X.; Wu, Y.; Yan, Z.; Han, J.; He, Z.; Drug Dev. Ind. Pharm. 2011, 37, 727 .

9. Lou, H.; Zhang, X.; Gao, L.; Feng, F.; Wang, J.; Wei, X.; Yu, Z.; Zhang, D.; Zhang, Q.; Int. J. Pharm. 2009, 379, 181.

10. Sigfridsson, K.; Lundqvist, A.J.; Strimfors, M.; Drug Dev. Ind. Pharm. 2009, 35, 1479

11. Ganta, S.; Paxton, J. W.; Baguley, B. C.; Garg, S.; Int. J. Pharm. 2009, $367,179$.

12. Rabinow, B.; Kipp, J.; Papadopoulos, P.; Wong, J.; Glosson, J.; Gass, J.; Sun, C. S.; Wielgos, T.; White, R.; Cook, C.; Barker, K.; Wood, K.; Int. J. Pharm. 2007, 339, 251.

13. Rabinow, B.; Discov. Med. 2005, 5, 74.

14. Marques, I.; Lopes, C. M.; Souto, E. B.; Rev. Facul. Ciências da Saúde 2009, 6, 60

15. Müller, R. H.; Becker, R.; Kruss, B.; Peters, K.; U.P. 5858410, 1999.

16. Shegokar, R.; Müller, R. H.; Int. J. Pharm. 2011, 399, 129.

17. Müller, R. H.; Gohla, S.; Keck, C. M.; Eur. J. Pharm. Biopharm. 2011, $78,1$.

18. Möschwitzer, J.; Müller, R. H.; International Meeting on Pharmaceutics, Biopharmaceutics and Pharmaceutical Technology, Nürnberg, Germany, 2004.

19. Verma, S.; Lan, Y.; Gokhale, R.; Burgess, D. J.; Int. J. Pharm. 2009, 377, 185.

20. Keck, C. M.; Müller, R. H.; Eur. J. Pharm. Biopharm. 2006, 62, 3.

21. Chen, H.; Khemtong, C.; Yang, X.; Chang, X.; Gao, J.; Drug Discov. Today 2010, 16, 354.

22. Gülsün, T.; Gürsoy, R. N.; Öner, L.; J. Pharm. Sci. 2009, 34, 55.

23. Xia, D.; Cui, F.; Piao, H.; Cun, D.; Piao, H.; Jiang, Y.; Ouyang, M.; Quan, P.; Pharm. Res. 2010, 27, 1965.

24. Petersen, R. D.; PCT/EP2007/009943, 2006

25. Gao, Y.; Li, Z.; Sun, M.; Guo, C.; Yu, A.; Xi, Y.; Cui, J.; Lou, H.; Zhai, G.; Drug Deliv. 2011, 18, 131.

26. Lou, H.; Gao, L.; Wei, X.; Zhang, Z.; Zheng, D.; Zhang, D, Zhang, X.; Li, Y.; Zhang, Q.; Colloids Surf., B 2011, 87, 319.

27. Sahoo, N. G.; Kakran, M.; Shaal, L. A.; Li, L.; Müller, R. H.; Pal, M.; Tan, L. P.; J. Pharm. Sci. 2011, 100, 2379.

28. Benita, S.; Levy, M. Y.; J. Pharm. Sci. 1993, 82, 1069.

29. Müller, R. H.; Jacobs, C.; Kayser, O. Em DissoCubes - a novel formulation for poorly soluble and poorly bioavailable drugs; Rathbone, M. J.; Hadgraft, J.; Roberts, M. S., eds.; Marcel Dekker: New York, 2003.

30. Kuo, F.; Subramanian, B.; Kotyla, T.; Wilson, T. A.; Yoganathan, S.; Nicolosi, R. J.; Int. J. Pharm. 2008, 363, 206.

31. Muchow, M.; Maincent, P.; Müller, R. H.; Drug Dev. Ind. Pharm. 2008, 34, 1394.

32. Wang, Y.; Liu, Z.; Zhang, D.; Gao, X.; Zhang, X.; Duan, C.; Jia, L.; Feng, F.; Huang, Y.; Shen, Y.; Zhang, Q.; Colloids Surf., B 2011, 83, 189.

33. Müller, R. H.; Krause, K.; Mäder, K.; WO/2001/003670, 2001.

34. Martins, S.; Tho, I.; Ferreira, D. C.; Souto, E. B.; Brandl, M.; Drug Dev. Ind. Pharm. 2011, 37, 815 .

35. Teeranachaideekul, V.; Junyaprasert, V. B.; Souto, E. B.; Müller, R. H.; Int. J. Pharm. 2008, 354, 227.

36. Sahoo, N. G.; Kakran, M.; Shaal, L. A.; Li, L.; Müller, R. H.; Pal, M.; Tan, L. P.; J. Pharm. Sci. 2011, 100, 2379. 
37. Buttle, I.; Dissertation, Freie Universität, Berlin, 2004.

38. Kipp, J. E. W.; Joseph, C. T.; Doty, M. J.; Rebbeck, C. L.; U.P. 6869617 , 2001.

39. Bawa, R.; Eur. J. Nanomedicine 2010, 3, 34.

40. Merisko-Liversidge, E.; Liversidge, G. G.; Cooper, E. R.; Eur. J. Pharm. Sci. 2003, 18, 113 .

41. Shegokar, R.; Singh, K. K.; Müller, R. H.; Int. J. Pharm. 2011, 416, 461.

42. Al Shaal, L.; Müller, R. H.; Shegokar, R.; Pharmazie 2010, 65, 877.
43. Han, X.; Ghoroi, C.; To, D.; Chen, Y.; Dave, R.; Int. J. Pharm. 2011, $415,185$.

44. Gu, C. H.; Grant, D. J.; J. Pharm. Sci. 2001, 90, 1277.

45. Wang, Y.; Li, X.; Wang, L.; Xu, Y.; Cheng, X.; Wei, P.; Int. J. Nanomedicine 2011, 6, 1497.

46. Li, W.; Yang, Y.; Tian, Y.; Xu, X.; Chen, Y.; Mu, L.; Zhang, Y.; Fang, L.; Int. J. Pharm. 2011, 408, 157. 